\title{
Brain Monoamine Oxidase A Activity Predicts Trait Aggression
}

\author{
Nelly Alia-Klein, ${ }^{1}$ Rita Z. Goldstein, ${ }^{1}$ Aarti Kriplani, ${ }^{1}$ Jean Logan, ${ }^{1}$ Dardo Tomasi, ${ }^{1}$ Benjamin Williams, ${ }^{2}$ Frank Telang ${ }^{4}$ \\ Elena Shumay, ${ }^{1}$ Anat Biegon, ${ }^{1}$ Ian W. Craig, ${ }^{2}$ Fritz Henn, ${ }^{1}$ Gene-Jack Wang, ${ }^{1,3}$ Nora D. Volkow, ${ }^{4}$ and Joanna S. Fowler ${ }^{1,3}$ \\ ${ }^{1}$ Medical Department, Brookhaven National Laboratory, Upton, New York 11973, ${ }^{2}$ King's College, London WC2R 2LS, United Kingdom, ${ }^{3}$ Mount Sinai \\ School of Medicine, New York, New York 10029, and ${ }^{4}$ National Institute on Alcohol Abuse and Alcoholism, Bethesda, Maryland 20892
}

The genetic deletion of monoamine oxidase A (MAO A), an enzyme that breaks down the monoamine neurotransmitters norepinephrine, serotonin, and dopamine, produces aggressive phenotypes across species. Therefore, a common polymorphism in the MAO A gene (MAOA, Mendelian Inheritance in Men database number 309850, referred to as high or low based on transcription in non-neuronal cells) has been investigated in a number of externalizing behavioral and clinical phenotypes. These studies provide evidence linking the low MAOA genotype and violent behavior but only through interaction with severe environmental stressors during childhood. Here, we hypothesized that in healthy adult males the gene product of MAO A in the brain, rather than the gene per se, would be associated with regulating the concentration of brain amines involved in trait aggression. Brain MAO A activity was measured in vivo in healthy nonsmoking men with positron emission tomography using a radioligand specific for MAO A (clorgyline labeled with carbon 11). Trait aggression was measured with the multidimensional personality questionnaire (MPQ). Here we report for the first time that brain MAO A correlates inversely with the MPQ trait measure of aggression (but not with other personality traits) such that the lower the MAO A activity in cortical and subcortical brain regions, the higher the self-reported aggression (in both MAOA genotype groups) contributing to more than one-third of the variability. Because trait aggression is a measure used to predict antisocial behavior, these results underscore the relevance of MAO A as a neurochemical substrate of aberrant aggression.

Key words: MAOA; aggression; violence; personality; clorgyline; human

\section{Introduction}

Trait aggression, defined as an enduring disposition toward physical assault, occurs at different levels in the general population and is an important predictor of future violence (Caspi et al., 2002; Moffitt et al., 2002). Therefore, understanding the neurochemical processes associated with trait aggression is vital to the control of maladaptive forms of this behavior. A potential neurochemical target is monoamine oxidase $\mathrm{A}$ (MAO A), an enzyme involved in metabolism of monoamines in brain and other organs (Shih and Thompson, 1999). MAO A catalytic activity resides in the mitochondria of presynaptic terminals in monoamine projection neurons throughout the brain, having a critical role in regulating the release and degradation of dopamine, serotonin (5-hydroxytryptamine) and norepinephrine (Westlund et al., 1993; Buckholtz and Meyer-Lindenberg, 2008).

\footnotetext{
Received Jan. 7, 2008; revised March 25, 2008; accepted March 25, 2008.

This work was accomplished at Brookhaven National Laboratory under contract DE-AC-298CH10886 with the U.S. Department of Energy and supported by its Office of Biological and Environmental Research, by National Institutes of Health General Clinical Research Center Grant M01RR10710, by National Institute on Drug Abuse Grant K05DA020001, and by National Alliance for Research on Schizophrenia and Depression. We thank David Alexoff, Donald Warner, Pauline Carter, Barbara Hubbard, Noelwah Netusil, Millard Jayne, Payton King, Hai-Dee Lee, Richard Ferrieri, Colleen Shea, Youwen Xu, Victor Garza, David Schlyer, and Michael Schueller for advice and assistance in various aspects of these studies.

Correspondence should be addressed to Dr. Nelly Alia-Klein or Dr. Joanna S. Fowler, Brookhaven National Laboratory-Medical, Building 490, Upton, NY 11973-5000. E-mail: nellyklein@bnl.gov or fowler@bnl.gov.

D01:10.1523/JNEUROSCI.0925-08.2008

Copyright $\odot 2008$ Society for Neuroscience $\quad$ 0270-6474/08/285099-06\$15.00/0
}

The clinical relevance of brain MAO A activity level (with its corresponding regulation of catecholaminergic activity) was highlighted by a recent study that documented a $34 \%$ elevation in brain MAO A activity in nonsmoking individuals with major depressive disorder (Meyer et al., 2006). High brain MAO A levels in depressed patients provide a neurobiological framework for the demonstrated efficacy of MAO inhibitors as antidepressants (Caldecott-Hazard and Schneider, 1992), and, because smokers have reduced brain MAO A (Fowler et al., 1996), it may also provide an explanation for the high rate of cigarette smoking in depression (Glassman et al., 2001). Conversely, low brain MAO A could be associated with aggression and its disorders (i.e., antisocial personality disorder). There is indirect evidence of highly aggressive behavior in men of one carrier family with a rare genetic mutation, resulting in deletion in the gene encoding for MAO A (known as MAOA) (Brunner et al., 1993). Subsequent studies similarly demonstrated an aggressive impulsive phenotype in MAOA knock-out mice (Cases et al., 1995; Shih, 2004) and in rat pups treated with clorgyline to inhibit MAO A and deprenyl to inhibit MAO B during gestation (Whitaker-Azmitia et al., 1994; Mejia et al., 2002). Recent cohort studies focusing on the prevalent functional polymorphism in the MAOA gene (low/ high, $\sim 40 \% / 60 \%$ in males) in human and nonhuman primates (Sabol et al., 1998; Newman et al., 2005) showed that the low MAOA genotype was associated with antisocial behavior and high self-reported trait aggression only with childhood exposure 
to severe maltreatment, thus highlighting the significance of the interplay between genes and environment (Kim-Cohen et al., 2006).

Still, important questions remain unanswered concerning the role of MAO A along intermediate phenotypes (MeyerLindenberg and Weinberger, 2006). Using positron emission tomography (PET) with $\left[{ }^{11} \mathrm{C}\right]$ clorgyline, we recently found no correspondence between the low and high MAOA genotype and brain MAO A activity in healthy males (Fowler et al., 2007) and others found no relationship in a postmortem sample (Balciuniene et al., 2002), nor were there any differences in resting brain glucose metabolism between the two genotypes (Alia-Klein et al., 2008). Particular voids in the literature pertain to levels of brain MAO A activity and its potential influence on individual differences in behavior and personality. Here, we advance our hypothesis that, if an internalizing disorder such as depression is associated with abnormally elevated brain MAO A activity, perhaps an externalizing behavior such as aggression in its escalated and enduring form would be associated with reductions in brain MAO A activity. This hypothesis has never been tested; if such a relationship exists, it would provide a mechanism for the study of trait aggression independent of the gene $\times$ environment effects of the MAOA genotype. Conceivably, the low MAO A genotype is involved in developmentally mediated susceptibility to aggression, whereas current brain MAO A activity provides a direct measurement of the enzyme activity and may therefore directly affect aggressive tendencies. In this study, we measured brain MAO A activity in manually drawn regions throughout the brain with PET and the radiotracer $\left[{ }^{11} \mathrm{C}\right.$ ]clorgyline (average dose, $6.2-$ $0.7 \mathrm{mCi}$; specific activity, $250 \mathrm{mCi} / \mathrm{micromol}$ ), which binds to catalytically active MAO A (Fowler et al., 1996), in 27 healthy participants that were also genotyped for MAOA polymorphism (Sabol et al., 1998). After the scan, personality was assessed with the multidimensional personality questionnaire (MPQ) (Tellegen and Waller, 1997).

\section{Materials and Methods}

Subjects. Participants in this study were 27 of the 38 participants in the previous study of brain MAO A activity versus genotype (Fowler et al., 2007) who also volunteered to complete the MPQ. All were healthy nonsmoking males recruited from newspaper advertisement and informed in accordance with the local Institutional Review Board. Nonsmoking status was obtained by self-report and substantiated through breath carbon monoxide measurement of $<6 \mathrm{ppm}$ (Cunnington and Hormbrey, 2002). Exclusion criteria were female gender, because of a 9:1 gender distribution of the phenotype (Caspi et al., 2002), current or past psychiatric or neurological disease, drug or alcohol abuse, history of cardiovascular or endocrinological disease, and current medical illness. Participants were genotyped for MAOA upstream variable number of tandem repeats (Sabol et al., 1998), resulting in 17 (63\%) participants with the high and 10 (37\%) with the low MAOA genotype. This genotype frequency distribution parallels previous studies (Sabol et al., 1998).

Personality. The MPQ is a self-report questionnaire that allows endorsing or rejecting 240 statements about how one perceives and describes himself (e.g., "I often lose my temper"). The MPQ is based on a contemporary and empirical structural model of dimensions (clusters) of personality traits (Church and Burke, 1994) related to aggression and other enduring patterns of behavior. Previous behavioral-genetic studies have established that the personality traits measured by the MPQ are heritable and stable, reflecting consistent behavior in the general population (Tellegen et al., 1988). Furthermore, several studies have demonstrated high reliability of the MPQ even in problem populations such as antisocial and violent men (Moffitt et al., 2002). Self-reported depression within 2 weeks of the scan was also assessed using the Beck Depression Inventory questionnaire (Beck et al., 1961).
Table 1. MAO A activity and trait aggression

\begin{tabular}{llll}
\hline & $r_{(25)}{ }^{a}$ & $r_{(8,15)}{ }^{b}$ & $t_{(7)}{ }^{c}$ \\
\hline Brain MA0 A cortical regions & & \\
Temporal & $-0.62^{* *}$ & $-0.60^{\text {n.s. }} /-0.65^{* *}$ & $-3.22^{*}$ \\
Occipital & $-0.63^{* *}$ & $-0.72^{*} /-0.56^{*}$ & $-3.18^{*}$ \\
Precuneus & $-0.64^{* *}$ & $-0.73^{*} /-0.54^{*}$ & $-4.24^{*}$ \\
Medial PFC & $-0.61^{* *}$ & $-0.65^{*} /-0.57^{*}$ & $-3.35^{*}$ \\
Frontal & $-0.54^{* *}$ & $-0.54^{\text {n.s. }} /-0.54^{*}$ & $-2.54^{*}$ \\
Subcortical regions & & & \\
Caudate & $-0.43^{*}$ & $-0.39^{\text {n.s. }} /-0.49^{*}$ & $-2.41^{*}$ \\
Amygdala & $-0.41^{\text {n.s. }}$ & $-0.53^{\text {n.s. }} /-0.38^{\text {n.s. }}$ & $-1.63^{\text {n.s. }}$ \\
Putamen & $-0.54^{* *}$ & $-0.53^{\text {n.s. }} /-0.55^{*}$ & $-3.41^{*}$ \\
Thalamus & $-0.56^{* *}$ & $-0.63^{*} /-0.48^{\text {n.s. }}$ & $-2.70^{*}$ \\
Pons & $-0.42^{*}$ & $-0.25^{\text {n.s. }} /-0.53^{*}$ & $-3.05^{*}$ \\
\hline
\end{tabular}

All tests were two-tailed. n.s, Nonsignificant. ${ }^{*} p<0.05 ;{ }^{* *} p<0.005$.

${ }^{a} r_{(25)}$ is the Pearson's correlation value between MAO A enzymatic activity $\left(\lambda k_{3}\right)$ in cortical and subcortical regions for all the participants $(n=27)$.

${ }^{b} r_{(8,15)}$ is the Pearson's correlations values for the low $(n=10)$ and high $(n=17)$ MAOA genotype groups.

${ }^{c} t_{(7)}$ is the $t$ test value of the independent samples comparison between aggressive $(n=4)$ and nonaggressive participants $(n=5)$ on MAO A activity in these regions.

${ }^{d}$ df for amygdala is 6 because of missing data as a result of technical difficulties.

Image acquisition. Brain MAO A activity was measured in each subject with $\left[{ }^{11} \mathrm{C}\right]$ clorgyline, which was shown to bind specifically to MAO A (Fowler et al., 1996). A whole-body PET scanner [Siemens (Munich, Germany) HR+; $4.5 \times 4.5 \times 4.8 \mathrm{~mm}$ at center of field of view] was used in three-dimensional dynamic acquisition mode, providing 63 contiguous planes of $2.4 \mathrm{~mm}$ each. The scanning period was $60 \mathrm{~min}$ with time frames of $6 \times 20 \mathrm{~s}, 4 \times 60 \mathrm{~s}, 2 \times 120 \mathrm{~s}$, and $10 \times$ 300 s. A transmission scan was obtained with a ${ }^{68} \mathrm{Ge}$ rotating rod source before the emission scan to correct for attenuation before the radiotracer injection. Arterial plasma samples were obtained over the time course of the study and were corrected for the presence of labeled metabolites (Alexoff et al., 1995).

MAO A activity was estimated by applying a three-compartment model to the $\left[{ }^{11} \mathrm{C}\right]$ clorgyline time-activity data from the brain and the arterial plasma. The $K_{1}$ is the plasma-to-brain transfer constant and is related to blood flow; $k_{2}$ is related to the transfer of tracer from brain to plasma. Finally, $\lambda k_{3}$ is an index of the amount of catalytically active MAO A (Fowler et al., 2001), where $\lambda$ is defined as $K_{1} / k_{2}$ and is independent of blood flow. It is important to note that the analyses herein are based on regions of interest (ROIs) that were manually drawn for each subject on an image formed by summing the time frames mentioned above. Bilateral ROIs were drawn (left and right averaged) in cortical regions [temporal, occipital, precuneus, and frontal cortices, as well as the medial prefrontal cortex (PFC)]; subcortical regions were drawn on pons and bilateral caudate, putamen, amygdala, and thalamus. These regions were then projected back onto the dynamic scans to obtain time-activity curves.

Statistical analyses. The relationship between the MPQ aggression scale and brain MAO A activity, measured in $\lambda k_{3}$ units, was tested with two-tailed Pearson's correlations, Bonferroni corrected for multiple comparisons (Stevens, 1992) $(p<0.05 / 10$ ROIs $=p<0.005)$ (Table 1) (see Fig. 1). To assess specificity to aggression versus other personality scales, we similarly tested the relationship between all the MPQ scales and MAO A brain activity in the frontal cortex $(p<$ $0.05 / 13$ scales $=p<0.004)$ (Table 2$)$. We chose the frontal cortex in Table 2 because of its relationship to aggression and poor inhibitory control (Meyer-Lindenberg et al., 2006). Note, however, that other regions produced similar results because MAO A activity in all regions was highly intercorrelated, making any emphasis on one brain region versus another difficult (Fowler et al., 1996). Table 2 also reports the relationships between the low and high MAO A genotype and personality that were tested with two-tailed independent samples $t$ tests (Table 2).

To validate a MAO A and aggression relationship and to compare whole-brain differences as a function of trait aggression, we used two-tailed independent samples $t$ tests. We compared participants 
Table 2. MPQ personality and $M A O A$ catalytic activity in the frontal cortex

\begin{tabular}{|c|c|c|c|c|}
\hline $\mathrm{MPQ}^{\text {scales }^{a}}$ & Description of high scorers & Mean $\pm S D^{b}$ & $r_{(25)}{ }^{c}$ & $t_{(25)}{ }^{d}$ \\
\hline Control (0-24) & Cautious, careful, reflective & $16.72 \pm 4.4$ & 0.00 & -0.30 \\
\hline Harm avoidance $(0-28)$ & Avoids danger, prefers safety even if tedious & $16.03 \pm 6.0$ & -0.05 & -0.36 \\
\hline Traditionalism $(0-27)$ & Conservative, needs predictable environment, high moral standards & $16.20 \pm 4.6$ & -0.07 & -0.41 \\
\hline Aggression $(0-20)$ & Willing to take advantage of others and cause them discomfort & $5.52 \pm 3.4$ & $-0.54^{* *}$ & 1.40 \\
\hline Alienation $(0-20)$ & Suspicious, feels mistreated, persecuted & $4.24 \pm 5.4$ & -0.05 & 1.30 \\
\hline Stress reaction $(0-14)$ & Nervous, sensitive, prone to overreact & $3.3 \pm 3.0$ & 0.02 & 1.74 \\
\hline Social closeness $(0-22)$ & Sociable, needs and likes people & $14.7 \pm 4.4$ & 0.00 & -0.16 \\
\hline Well-being $(0-11)$ & Happy, cheerful, feels good about oneself and the future & $9.2 \pm 2.4$ & 0.04 & -0.33 \\
\hline Achievement $(0-21)$ & Works hard, enjoys demanding projects, persists for long hours & $13.1 \pm 4.0$ & -0.02 & -0.69 \\
\hline Social potency $(0-26)$ & Forceful, decisive, influences others, enjoys leadership roles & $14.0 \pm 5.9$ & -0.25 & 1.92 \\
\hline Constraint & Control + harm avoidance + traditionalism & $49.2 \pm 11.3$ & -0.02 & -0.42 \\
\hline Negative emotionality & Aggression + alienation + stress reaction & $12.5 \pm 9.0$ & -0.18 & 2.00 \\
\hline Positive emotionality & Social closeness + well-being + achievement + social potency & $51.2 \pm 10.9$ & -0.15 & 0.70 \\
\hline
\end{tabular}

All tests were two-tailed. ${ }^{* *} p<0.003$.

${ }^{a}$ In parentheses, we provide the range of scores for the MPQ scales; the last three are personality factors empirically constructed from combination of some of the scales.

${ }^{b}$ Means and SD are provided for all participants $(n=27)$.

${ }^{c} r_{(25)}$ is the Pearson's correlation value for each of the scales with MAO A enzymatic activity $\left(\lambda k_{3}\right)$ in the frontal cortex for all the participants.

${ }^{d} t_{(25)}$ is the $t$ test value of the independent samples comparison between low $(n=10)$ and high $(n=17)$ MAO A genotype groups.

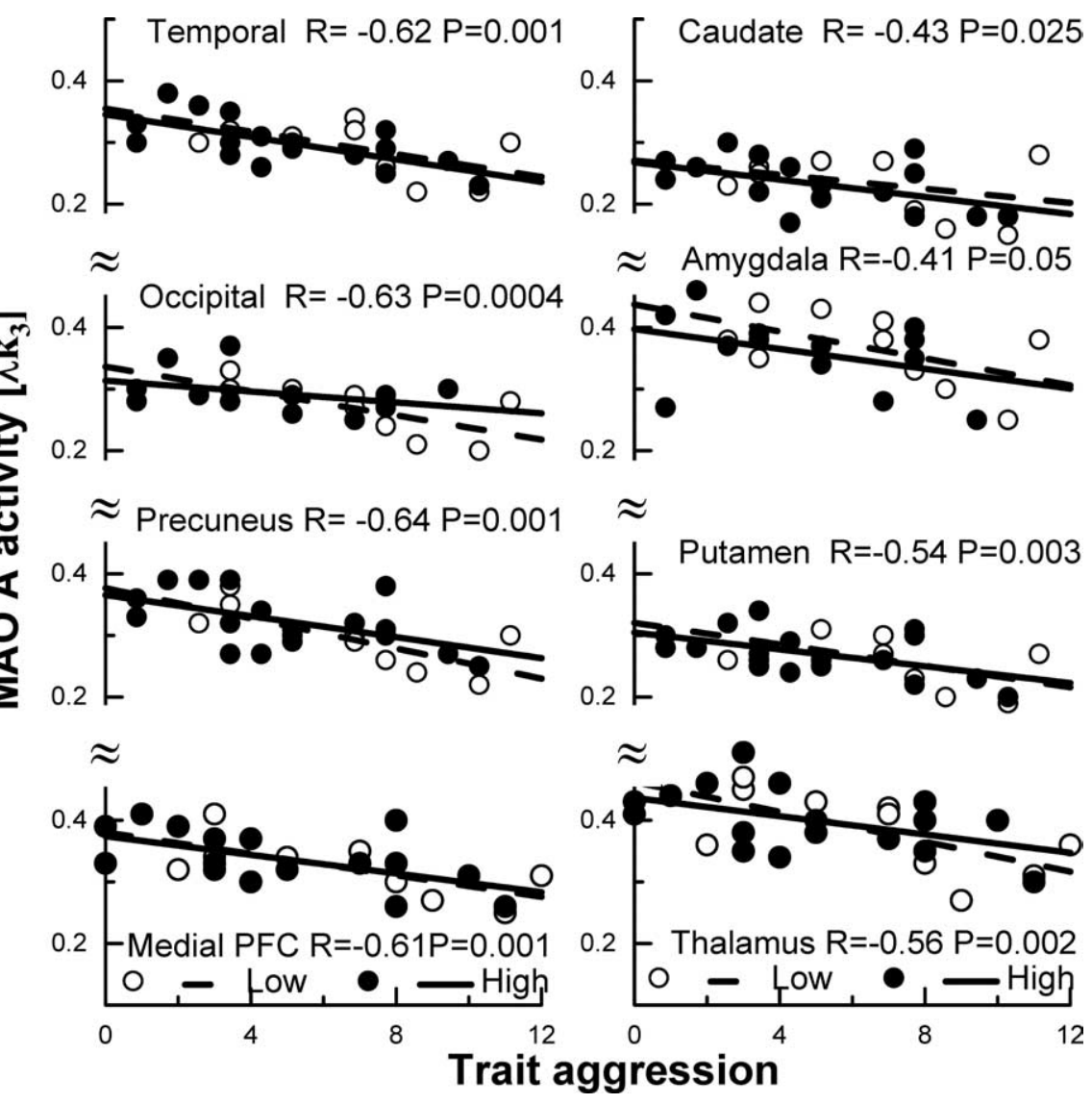

Figure 1. Trait aggression and brain MAOA activity in cortical regions (left) and in subcortical regions (right). The $r$ and $p$ values at the top of each scatter plot represent the correlation value and significance for the whole population ( $d f=25)$. The units of $M A 0$ A activity, $\lambda k_{3}$, are an index of the amount of catalytically active MAO A. Data from the two hemispheres was combined by averaging the right and the left sides of each region. White circles denote low MAO A genotype $(n=10)$, and black circles denote high MAO A genotype $(n=17)$.

who reported trait aggression at $1 \mathrm{SD}$ above the mean of the group to participants that were $1 \mathrm{SD}$ below the mean (aggressive and nonaggressive, respectively) (see Fig. 2 for whole-brain analyses and Fig. 3 for ROIs). For these validation analyses, we used $p<0.05$, uncorrected because of our a priori hypothesis that brain MAO A activity will correlate with trait aggression; thus, aggressive subjects should have less brain MAO A binding activity than nonaggressive subjects.
Note that the images in Figure 2 were normalized to the SPM (Wellcome Department of Cognitive Neurology, University College London, London, UK) PET template so that the individual frames could be averaged before forming the parametric image (Reimold et al., 2004). It was necessary to average the frame data to increase the signal-to-noise ratio. In some individuals with low blood flow (and in some regions of almost all participants), estimates of the model parameter associated with MAO A were very sensitive to noise such that reliable estimates could not be obtained without averaging; here, we used discrete frame averaging that increases the signal sufficiently to overcome this problem. Thus, an averaged image for each group allowed us to study the differences between the aggressive and nonaggressive groups.

\section{Results}

The participants' aggression scores ranged from 0 to 12 (mean $\pm \mathrm{SD}, 5.52 \pm 3.44$ ); brain MAO A activity ranged from 0.27 to $0.51 \mathrm{cc}_{\text {brain }}\left(\mathrm{ml}_{\text {plasma }}\right)^{-1}(0.39 \pm 0.06)$ in thalamus, which shows the highest binding. Other regions produced similar results, and both brain and behavior measurements were comparable with others (Fowler et al., 1996; Tellegen and Waller, 1997).

Brain MAO A correlated inversely with the MPQ trait measure of aggression such that the lower the brain MAO A activity, the higher the self-reported aggression across both genotype groups and throughout the brain (Table 1). Figure 1 shows simple regressions plotting MAO A activity in our bilateral ROIs in cortical regions (temporal, occipital, precuneus, and medial prefrontal cortices) and subcortical regions (caudate, putamen, amygdala, and thalamus) with the MPQ aggression scores. Comparing aggressive and nonaggressive participants, Figure 2 shows the reduced brain MAO A activity in those participants who reported relatively high trait aggres- 


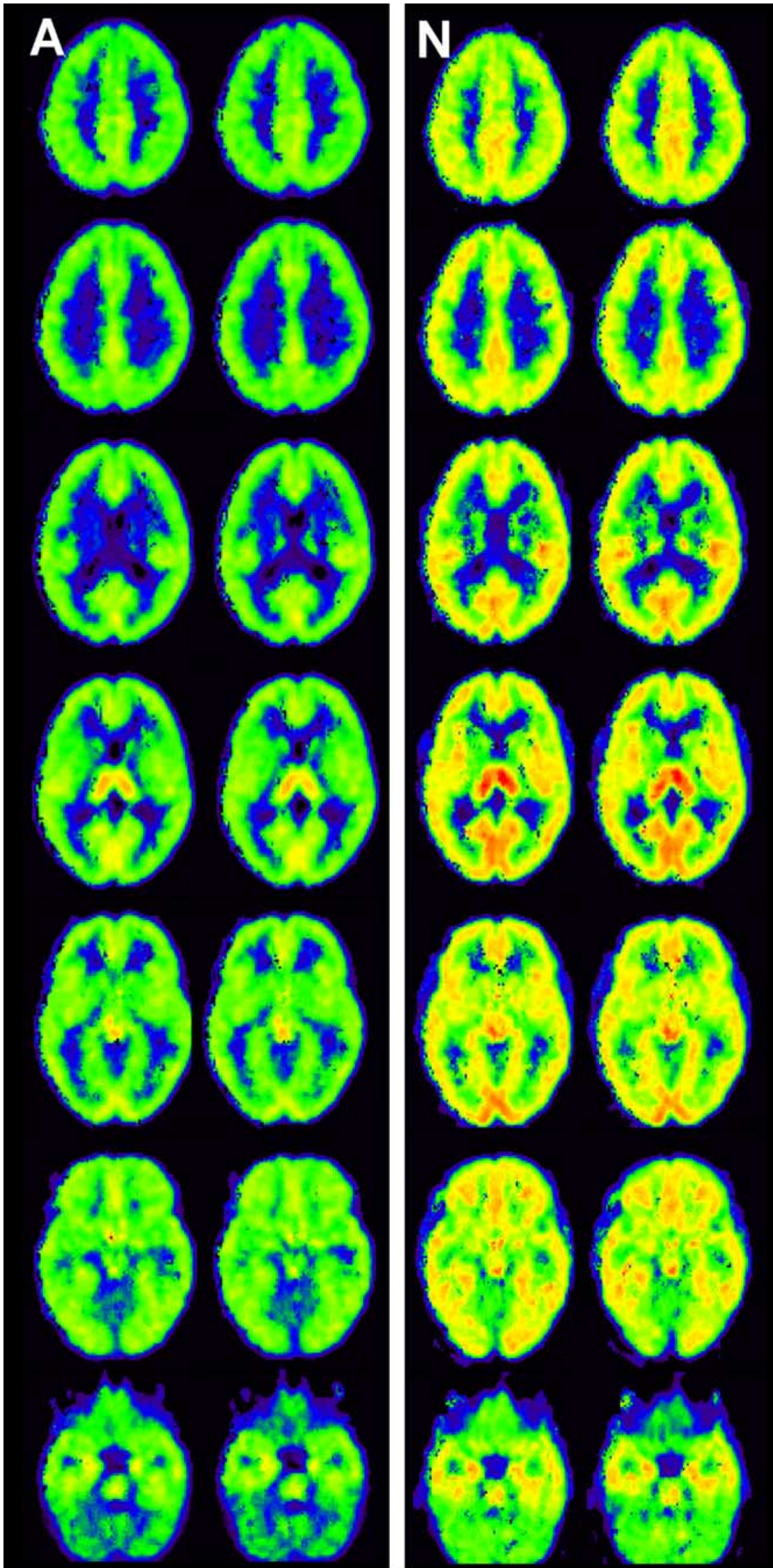

Figure 2. Brain MAO A activity in aggressive and in nonaggressive participants. On the left vertical panel marked as " $\mathrm{A}$ " is averaged images of participants who endorsed 10 or more aggression questions $(n=4)$. On the right is an averaged image of participants who endorsed two or less aggression questions ( $n=5$; marked as " $\mathrm{N}$ ") based on 1 SD above or below the aggression mean for this sample. The images are presented in continuous axial slices throughout most of the brain from superior to inferior sion compared with nonaggressive participants, a contrast that displays a visible difference in MAO A activity throughout the brain. Using ROIs, these differences were statistically significant between the groups (Fig. 3).

Importantly, the findings were unique to trait aggression. Brain MAO A did not correlate with any of the other MPQ scales ( $p=0.11-0.97)$. Similarly, brain MAO A was not related to self-reported depression within 2 weeks of the PET scan $(r=$ $-0.002 ; p=0.99$ ). As can be surmised from Figure 1, $A$ and $B$, the low and high MAOA genotype groups had similar correlations with trait aggression, and there were no differences in any of the MPQ scales as a function of MAOA genotype (Table 2).

\section{Discussion}

The MPQ aggression scale measures attitudes, values, and beliefs that are consistent with approval of the use of physical violence. Longitudinal research shows that the MPQ aggression scale empirically predicts future conviction for violent crime (Moffitt et al., 2002) such as physical assault (Craig, 2007). Here we report for the first time that the lower the MAO A activity in cortical and subcortical brain regions, the higher the self-reported aggression, contributing to more than one-third of the variability among a sample of 27 healthy men.

Because the MAO A regional activity is highly intercorrelated, we cannot make claims about the specificity in trait aggression of certain brain regions, although it appears that the correlations are stronger in cortical regions (Fig. 1). These results are consistent with previous imaging studies of MAO A and B. Although both subtypes are highest in the thalamus, MAO A is higher in cortical regions than MAO B (Fowler et al., 2005). It is important to remember that,

planes for both participant groups. To increase the signal-tonoise in these images, we used discrete frame averaging of images from each subject before the formation of parametric images (Reimold et al., 2004). The dynamic images were normalized to the SPM PET template (http://www.fil.ion. ucl.ac.uk/spm/). Average dynamic images weighted by the radiotracer dose were formed for each group. Average blood input functions were also formed from data of the participants in each group. Parametric images of model parameter $\lambda k_{3}$ were constructed using the method of Feng et al. (1996) for a twotissue compartment irreversible model. A small amount of averaging to increase the signal-to-noise was allowed for local pixels using a method described previously (Logan et al., 2002). The average number of pixels grouped was on the order of 4. 


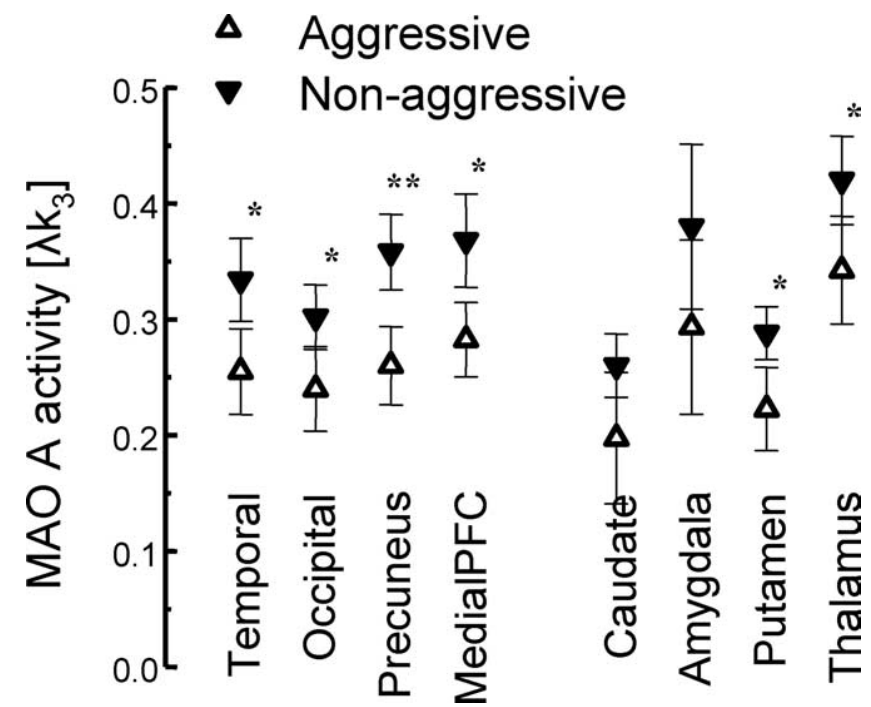

Figure 3. The mean $\pm S D$ of brain MAOA activity, $\lambda k_{3}$, in aggressive versus nonaggressive participants in cortical and subcortical regions. White triangles represent the mean for the aggressive group $(n=4)$, and black triangles represent the nonaggressive group $(n=5) .{ }^{*} p<$ $0.05 ;{ }^{* *} p<0.01$.

to avoid the confounding effects of cigarette smoking on reducing brain MAO A activity (Fowler et al., 1996), this sample comprised nonsmoking participants. Indeed, the potential multifactorial relationship between prenatal smoking exposure and the neurochemistry underlying externalizing behaviors [e.g., impulsivity and aggression (Maughan et al., 2004)] remains to be studied. In considering the clinical implications of our findings, it is important to note that, because MAO A enzymatic activity is responsible for the regulation of central serotonergic activity in terms of availability and subsequent extrasynaptic degradation (for a recent review, see Buckholtz and Meyer-Lindenberg, 2008), it would seemingly follow that having low MAO A activity will result in high serotonin levels. Indeed, MAO A knock-out mice have high circulating serotonin (Cases et al., 1995); however, when serotonin metabolites are examined in aggressive humans (Moffitt et al., 1998) as well as aggressive rhesus monkeys (Howell et al., 2007), they are found to be low. This difference is possibly attributable to compensatory mechanisms that primates may have developed to maintain control of high serotonin levels that could be neurotoxic (Baumgarten and Lachenmayer, 2004). Future studies should test the interaction of serotonin and MAO A in the human brain in vivo to understand their functional role in trait aggression.

Behavioral-genetic longitudinal studies have established that the low MAOA genotype predicts high trait aggression (notably using the MPQ) in men who were severely maltreated at childhood (Caspi et al., 2002). In addition, the low MAOA genotype in healthy adults from the general population has been associated with morphological and functional changes in the amygdala (a brain region involved in expression of emotions), medial PFC (a region involved with emotional control), and in other regions that could underlie vulnerability for the expression of aggression (Meyer-Lindenberg et al., 2006). Yet we found that neither trait aggression nor brain MAO A activity differed as a function of the MAOA genotype in the current sample, suggesting that these gene-behavior and gene-brain relationships may be developmentally mediated (Fowler et al., 2007). It is hypothesized that, at sensitive stages during early development, changes in MAO A balance triggered by a severe environmental assault could disrupt monoamine-mediated brain development leading toward the expression of an aggressive phenotype. Indeed, psychosocial stress during early development is thought to play a significant role in modulating prefrontal and subcortical MAO A activity and susceptibility for aggression and impulsivity (Nelson and Trainor, 2007). Because in the current study we did not assess environmental factors such as childhood stress, we cannot rule out the potential interactive contribution of the MAOA genotype to trait aggression. Clearly, the degree of exposure to stress would be an important variable to measure in future imaging studies.

Considering the multifactor architecture of human aggression (Buckholtz and Meyer-Lindenberg, 2008), MAO A activity in the brain provides a distinct and biological intermediate phenotype that is affected by various modifiers throughout life and is not limited by the specific polymorphism in one gene. Thus, our findings provide evidence that brain MAO A activity is a tractable target more immediate to the behavioral phenotype than the MAOA genotype stratification in healthy men. An approximate $15 \%$ reduction in brain MAO A is associated with elevated trait aggression (Figs. 2, 3), or, put differently, brain MAO A explained $>30 \%$ of the variability in trait aggression among these men. These findings underscore the involvement of MAO A enzymatic activity as a neurochemical target with clinical implications for the treatment of aberrant aggression.

\section{References}

Alexoff DL, Shea C, Fowler JS, King P, Gatley SJ, Schlyer DJ, Wolf AP (1995) Plasma input function determination for PET using a commercial laboratory robot. Nucl Med Biol 22:893-904.

Alia-Klein N, Kriplani A, Pradhan K, Yemin Ma J, Logan J, Williams B, Craig IW, Telang F, Tomasi D, Goldstein RZ, Wang GJ, Vokow ND, Fowler JS (2008) The MAO A genotype does not modulate resting brain metabolism in adults. Psychiatry Res Neuroimaging, in press.

Balciuniene J, Emilsson L, Oreland L, Pettersson U, Jazin E (2002) Investigation of the functional effect of monoamine oxidase polymorphisms in human brain. Hum Genet 110:1-7.

Baumgarten HG, Lachenmayer L (2004) Serotonin neurotoxins-past and present. Neurotox Res 6:589-614.

Beck AT, Ward CH, Mendelson M, Mock J, Erbaugh J (1961) An inventory for measuring depression. Arch Gen Psychiatry 4:561-571.

Brunner HG, Nelen M, Breakefield XO, Ropers HH, van Oost BA (1993) Abnormal behavior associated with a point mutation in the structural gene for monoamine oxidase A. Science 262:578-580.

Buckholtz JW, Meyer-Lindenberg A (2008) MAOA and the neurogenetic architecture of human aggression. Trends Neurosci 31:120-129.

Caldecott-Hazard S, Schneider LS (1992) Clinical and biochemical aspects of depressive disorders. III. Treatment and controversies. Synapse 10:141-168.

Cases O, Seif I, Grimsby J, Gaspar P, Chen K, Pournin S, Muller U, Aguet M, Babinet C, Shih JC (1995) Aggressive behavior and altered amounts of brain serotonin and norepinephrine in mice lacking MAOA. Science 268:1763-1766.

Caspi A, McClay J, Moffitt TE, Mill J, Martin J, Craig IW, Taylor A, Poulton R (2002) Role of genotype in the cycle of violence in maltreated children. Science 297:851-854.

Church AT, Burke PJ (1994) Exploratory and confirmatory tests of the big five and Tellegen's three- and four-dimensional models. J Pers Soc Psychol 66:93-114

Craig IW (2007) The importance of stress and genetic variation in human aggression. BioEssays 29:227-236.

Cunnington AJ, Hormbrey P (2002) Breath analysis to detect recent exposure to carbon monoxide. Postgrad Med J 78:233-237.

Feng D, Li X, Huang SC (1996) A new double modeling approach for dynamic cardiac PET studies using noise and spillover contaminated LV measurements. IEEE Trans Biomed Eng 43:319-327.

Fowler JS, Volkow ND, Wang GJ, Pappas N, Logan J, Shea C, Alexoff D, MacGregor RR, Schlyer DJ, Zezulkova I, Wolf AP (1996) Brain mono- 
amine oxidase A inhibition in cigarette smokers. Proc Natl Acad Sci USA 93:14065-14069.

Fowler JS, Logan J, Ding YS, Franceschi D, Wang GJ, Volkow ND, Pappas N, Schlyer D, Gatley SJ, Alexoff D, Felder C, Biegon A, Zhu W (2001) NonMAO A binding of clorgyline in white matter in human brain. J Neurochem 79:1039-1046.

Fowler JS, Logan J, Volkow ND, Wang GJ (2005) Translational neuroimaging: positron emission tomography studies of monoamine oxidase. Mol Imaging Biol 7:377-387.

Fowler JS, Alia-Klein N, Kriplani A, Logan J, Williams B, Zhu W, Craig IW, Telang F, Goldstein R, Volkow ND, Vaska P, Wang GJ (2007) Evidence that brain MAO A activity does not correspond to MAO A genotype in healthy male subjects. Biol Psychiatry 62:355-358.

Glassman AH, Covey LS, Stetner F, Rivelli S (2001) Smoking cessation and the course of major depression: a follow-up study. Lancet 357:1929-1932.

Howell S, Westergaard G, Hoos B, Chavanne TJ, Shoaf SE, Cleveland A, Snoy PJ, Suomi SJ, Dee Higley J (2007) Serotonergic influences on life-history outcomes in free-ranging male rhesus macaques. Am J Primatol 69:851-865.

Kim-Cohen J, Caspi A, Taylor A, Williams B, Newcombe R, Craig IW, Moffitt TE (2006) MAOA, maltreatment, and gene-environment interaction predicting children's mental health: new evidence and a meta-analysis. Mol Psychiatry 11:903-913.

Logan J, Fowler JS, Ding YS, Franceschi D, Wang GJ, Volkow ND, Felder C, Alexoff D (2002) Strategy for the formation of parametric images under conditions of low injected radioactivity applied to PET studies with the irreversible monoamine oxidase A tracers $\left[{ }^{11} \mathrm{C}\right]$ clorgyline and deuterium-substituted $\left[{ }^{11} \mathrm{C}\right]$ clorgyline. J Cereb Blood Flow Metab 22:1367-1376.

Maughan B, Taylor A, Caspi A, Moffitt TE (2004) Prenatal smoking and early childhood conduct problems: testing genetic and environmental explanations of the association. Arch Gen Psychiatry 61:836-843.

Mejia JM, Ervin FR, Baker GB, Palmour RM (2002) Monoamine oxidase inhibition during brain development induces pathological aggressive behavior in mice. Biol Psychiatry 52:811-821.

Meyer JH, Ginovart N, Boovariwala A, Sagrati S, Hussey D, Garcia A, Young T, Praschak-Rieder N, Wilson AA, Houle S (2006) Elevated monoamine oxidase a levels in the brain: an explanation for the monoamine imbalance of major depression. Arch Gen Psychiatry 63:1209-1216.

Meyer-Lindenberg A, Weinberger DR (2006) Intermediate phenotypes and genetic mechanisms of psychiatric disorders. Nat Rev Neurosci $7: 818-827$
Meyer-Lindenberg A, Buckholtz JW, Kolachana B, R Hariri A, Pezawas L, Blasi G, Wabnitz A, Honea R, Verchinski B, Callicott JH, Egan M, Mattay V, Weinberger DR (2006) Neural mechanisms of genetic risk for impulsivity and violence in humans. Proc Natl Acad Sci USA 103:6269-6274

Moffitt TE, Brammer GL, Caspi A, Fawcett P, Raleigh M, Yuwiler A, Silva PA (1998) Whole blood serotonin relates to violence in an epidemiological study. Biol Psychiatry 43:446-457.

Moffitt TE, Caspi A, Harrington H, Milne BJ (2002) Males on the lifecourse-persistent and adolescence-limited antisocial pathways: follow-up at age 26 years. Dev Psychopathol 14:179-207.

Nelson RJ, Trainor BC (2007) Neural mechanisms of aggression. Nat Rev Neurosci 8:536-546.

Newman TK, Syagailo YV, Barr CS, Wendland JR, Champoux M, Graessle M, Suomi SJ, Higley JD, Lesch KP (2005) Monoamine oxidase A gene promoter variation and rearing experience influences aggressive behavior in rhesus monkeys. Biol Psychiatry 57:167-172.

Reimold M, Mueller-Schauenburg W, Becker GA, Reischl G, Dohmen BM, Bares R (2004) Non-invasive assessment of distribution volume ratios and binding potential: tissue heterogeneity and interindividually averaged time-activity curves. Eur J Nucl Med Mol Imaging 31:564-577.

Sabol SZ, Hu S, Hamer D (1998) A functional polymorphism in the monoamine oxidase A gene promoter. Hum Genet 103:273-279.

Shih JC (2004) Cloning, after cloning, knock-out mice, and physiological functions of MAO A and B. Neurotoxicology 25:21-30.

Shih JC, Thompson RF (1999) Monoamine oxidase in neuropsychiatry and behavior. Am J Hum Genet 65:593-598.

Stevens J (1992) Applied multivariate statistics for the social sciences, Ed 2. Hillsdale, NJ: Erlbaum.

Tellegen A, Waller NG (1997) Exploring personality through test construction: development of the multidimensional personality questionnaire. In: Personality measures: development and evaluation (Briggs SR, Cheek JM, eds). Greenwich, CT: JAI.

Tellegen A, Lykken DT, Bouchard Jr TJ, Wilcox KJ, Segal NL, Rich S (1988) Personality similarity in twins reared apart and together. J Pers Soc Psychol 54:1031-1039.

Westlund KN, Krakower TJ, Kwan SW, Abell CW (1993) Intracellular distribution of monoamine oxidase $\mathrm{A}$ in selected regions of rat and monkey brain and spinal cord. Brain Res 612:221-230.

Whitaker-Azmitia PM, Zhang X, Clarke C (1994) Effects of gestational exposure to monoamine oxidase inhibitors in rats: preliminary behavioral and neurochemical studies. Neuropsychopharmacology 11:125132. 